\title{
Simplified equation for determining proper depth of peripherally inserted central catheter in relation to anatomical landmarks
}

\author{
Sang-Soo Kang ${ }^{1}$, Yang-Sik Shin ${ }^{2}$, Seon-Yi Lee ${ }^{2}$, and Hyunzu Kim ${ }^{3}$ \\ Department of Anesthesiology and Pain Medicine, ${ }^{1}$ Kangdong Sacred Heart Hospital, Hallym University College \\ of Medicine, Seoul, ${ }^{2}$ CHA Bundang Hospital, CHA University College of Medicine, Seongnam, ${ }^{3}$ Inha University \\ College of Medicine, Incheon, Korea
}

Background: The aim of this study was to develop a formula guiding the peripherally inserted central catheter (PICC) tip placement based on anatomical landmarks such as the upper arm, clavicle, and sternum as well as the patient's height, weight, and body mass index.

Methods: Fifty-five patients who were scheduled to have PICCs were included in the study. We measured four distances along the passage of the PICC, which were as follows; the tip of the third finger to the middle of the elbow crease (Distance A), the middle of the elbow crease to the acromion process (Distance B), the acromion process to the sternal head of the clavicle (Distance C), and the sternal head of the clavicle to the end of the xiphoid process (Distance D). The lengths from the elbow creases to their carina bifurcations as determined by fluoroscopy during PICC insertions were recorded and used as reference.

Results: The formula for determining PICC depth based on the four distances was determined by regression analysis. The optimal formula was determined to be $25.3+0.5 \times($ Distance $C)+0.6 \times($ Distance $D)$ which yielded an $\mathrm{R}^{2}$ value of 0.3 . Conclusions: The formula proposed for proper depth of the adult, $25.0+0.5 \times$ (clavicle length) $+0.6 \times$ (sternum length) for PICC insertion can be used to place the tip at the carina bifurcation level. The distance from elbow crease to catheter insertion point should be added to the length generated by this formula.

Keywords: Anatomic landmarks; Fluoroscopy; Peripherally inserted central catheter line insertion; Regression analysis.

Corresponding author: Hyunzu Kim, M.D., Ph.D.

Department of Anesthesiology and Pain Medicine, Inha University College of Medicine, 27, Inhang-ro, Jung-gu, Incheon 22332, Korea

Tel: 82-32-890-3968, Fax: 82-32-991-2476

Email: aneshyunzu@gmail.com

ORCID: https://orcid.org/0000-0002-0625-6942

Received: August 2, 2017.

Revised: August 11, 2017 (1st); August 22, 2017 (2nd).

Accepted: August 22, 2017.

Korean J Anesthesiol 2018 August 71(4): 300-304

https://doi.org/10.4097/kja.d.18.27185

\section{Introduction}

Peripherally inserted central catheter (PICC) is a kind of a kind of central venous catheter, usually inserted in the upper extremity to the superior vena cava. PICCs can provide reliable central venous pressure measurement and vascular access for vasoactive drug and fluid administration [1]. Moreover, PICCs can be kept for long durations and so can be used for continuous management such as antibiotic therapy, sustained total parenteral nutrition, or chemotherapy. For these reasons, PICCs have become one of the most frequently used central venous catheters in intensive care units [2].

As the central catheter tip must be positioned at the ca-

(c) This is an open-access article distributed under the terms of the Creative Commons Attribution Non-Commercial License (http://creativecommons.org/ licenses/by-nc/4.0/), which permits unrestricted non-commercial use, distribution, and reproduction in any medium, provided the original work is properly cited. 
voatrial junction, many reports have been published on how to determine the correct depth for central venous catheterization in adult [3-5] and pediatric [6-8] patients. Inappropriate catheter tip positioning can result in inaccurate hemodynamic monitoring and increase in complications that may arise from misplacing the tip of the catheter in the right atrium such as arrhythmia, thrombosis, cardiac perforation, and tamponade [9-13]. Despite these risks, there is no widely accepted standard formula estimating the depth of PICC, so individual depth is usually determined by chest X-ray afterwards [14,15]. Given the risks, the purpose of this study was to develop a formula guiding the PICC tip placement based on anatomical landmarks such as the upper arm, clavicle, and sternum as well as the patient's height, weight, and body mass index.

\section{Materials and Methods}

This study was registered with the Clinical Research Information Service after it was approved by the Institutional Review Board of our hospital. The patient recruitment period was one year. Patients who were scheduled for PICC insertion were asked for informed consent. Patients who were excluded were those with a body mass index greater than $30 \mathrm{~kg} / \mathrm{m}^{2}$, history of heart or great vessel surgery, trauma, or congenital anomalies. Fifty-five patients were enrolled in the study, and the data on age, gender, weight, and height were collected from each of the subjects.

PICC insertion was always performed by an expert anesthesiologist following evidence-based institutional protocols in an operating room where fluoroscopy could be used. The patients were laid supine on the angiographic table with their right arm extended to the side spread straight. Before the anesthesiologist inserted the PICC, five points along the passage of PICC were marked on the patient's skin and the distances between each point were measured (Fig. 1). Distance A was from the tip of the 3rd finger to the middle of the elbow crease. Distance B was from the middle of the elbow crease to the most prominent point of the acromion process. Distance $\mathrm{C}$ was from the most prominent point of the acromion process to the sternal head of the clavicle. Distance D was from the sternal head of the clavicle to the end of the xiphoid process.

The patients were breathing spontaneously during the procedure and no attempt was made to control respiration. Preprocedure ultrasounds were performed to identify whether the appropriately sized vessel was the cephalic or basilic vein and ensure that it was clot-free. A tourniquet was placed around the upper right arm and tightened by an assistant taking maximal sterile barrier precautions. After $3 \mathrm{ml} \mathrm{2 \%}$ lidocaine was administered subcutaneously, the vein was percutaneously accessed guided by ultrasonography. Then the vein was accessed using a modified Seldinger technique with a regular hypodermic needle, an intravenous cannula. A guide wire was threaded into the cannula and not advanced past the shoulder. A nick was then made in the skin beside the guide wire and an introducer sheath with a dilator was inserted over the guide wire. The guide wire and dilator were then removed and the catheter was advanced through the introducer sheath. The PICC's tip position was confirmed to be at the carina bifurcation using fluoroscopy [16,17]. After the peel-away sheath was removed, blood was aspirated from the catheter and fluids were infused to keep the lumen patent. The catheter was secured in place with sutures and a sterile dressing was applied to the site. Actual PICC length was recorded by measuring the distance from the insertion site to the carina bifurcation. The actual length, which was the distance from insertion site to the carina bifurcation level, was corrected and revised by accounting for the distance between the elbow crease and the skin insertion site. The revised length defined the depth of PICC to be used as a reference to the tested formula, which was the primary endpoint in this study.

Regarding the patient's height, plots of the revised depth of PICC against Distance A, B, C, and D were generated, and linear regression analysis was performed to calculate the coefficient of determination $\left(\mathrm{R}^{2}\right)$. To select the optimal variables, we analyzed in three steps. First, diverse univariate or multivariate linear regression models were created with variables. Second, multiple models were constructed using the four distances as variables selected by forward, backward, and stepwise methods. Third, we verified the model by reducing the variables that was statistically significant. After performing the three steps, the optimal regression equation was determined on the revised PICC depth

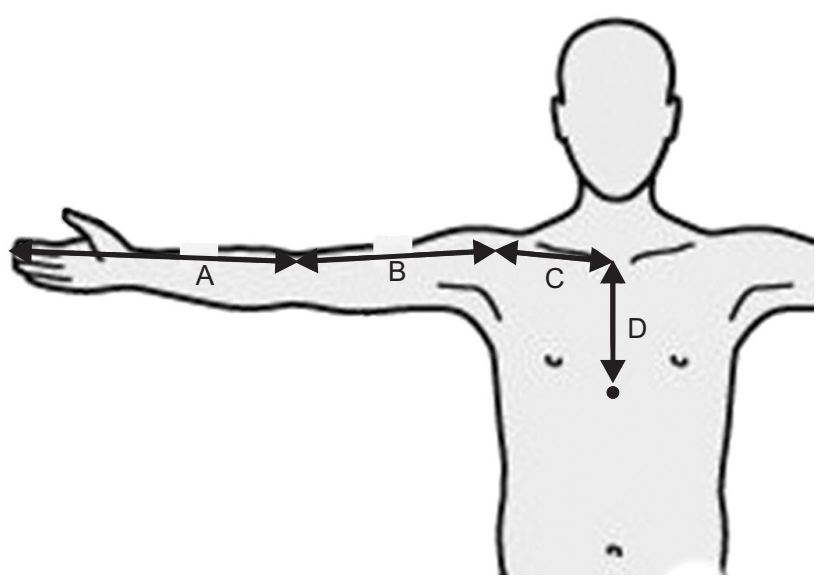

Fig. 1. Five points and four distances to be measured. The four distances are the tip of the third finger to the middle of the elbow crease (Distance A), the middle of the elbow crease to the acromion process (Distance B), the acromion process to the sternal head of the clavicle (Distance C), and the sternal head of the clavicle to the end of the xiphoid process (Distance D). 
Table 1. Demographic Data of Participants

\begin{tabular}{lc}
\hline & Values \\
\hline Age $(\mathrm{yr})$ & $65.9 \pm 15.3$ \\
Gender $(\mathrm{M} / \mathrm{F})$ & $27 / 27$ \\
Weight $(\mathrm{kg})$ & $57.4 \pm 12.5$ \\
Body mass index $\left(\mathrm{kg} / \mathrm{m}^{2}\right)$ & $22.6 \pm 4.9$ \\
\hline
\end{tabular}

Values are expressed as mean \pm SD or number of patients.

Table 2. Correlation Coefficients between PICC Depth and Patient's Height, Distance A, B, C, D

\begin{tabular}{lccr}
\hline & Values & $\begin{array}{c}\text { Correlation coefficient } \\
\text { against the depth of } \\
\text { PICC }\end{array}$ & P value \\
\hline Height & $157.2 \pm 21.9$ & 0.329 & 0.015 \\
Distance A & $40.4 \pm 2.7$ & 0.321 & 0.018 \\
Distance B & $29.4 \pm 2.7$ & 0.067 & 0.630 \\
Distance C & $19.1 \pm 2.3$ & 0.483 & $<0.001$ \\
Distance D & $18.4 \pm 2.3$ & 0.506 & $<0.001$ \\
\hline
\end{tabular}

Values are expressed as mean \pm SD. PICC: peripherally inserted central catheter. Distance A: tip of 3rd finger - midline of elbow crease, Distance B: midline of elbow crease - acromion process, Distance C: acromion process - sternal head of clavicle, Distance D: sternal head of clavicle - end of xiphoid process.

and the variables (distances). Statistical analysis was performed using SAS version 9.4 software (SAS Institute Inc., USA) and a P value of 0.05 was considered as statistically significant.

\section{Results}

All fifty-five patients completed the study. The demographic data of these patients are presented in Table 1.

PICC depth was positively and linearly correlated with the patient's height and 4 distances. Table 2 shows the correlation coefficients and results of univariate linear regression analysis between the PICC depth, the patient's height, and 4 distances.

First, eleven univariate or multivariate linear regression models were created using the height and four distances as variables. The root mean square error, adjusted R-squared, and Akaike's information criterion of each model were compared. The model that contained Distances C and D was the model with the best fit. Second, the models with Distances C and D produced the best fit using all three methods. Third, a general linear F-test was conducted to confirm whether the conversion from a full model, which used all variables, to a reduced model, which used selected variables, still produced statistically significant results with $\mathrm{P}$ values $>0.05$. After three simplification steps were conducted, only the model using Distances $\mathrm{C}$ and $\mathrm{D}$ as variables remained. In the next step, there were no other models that could be used to simplify the model by reducing the number of variables used,

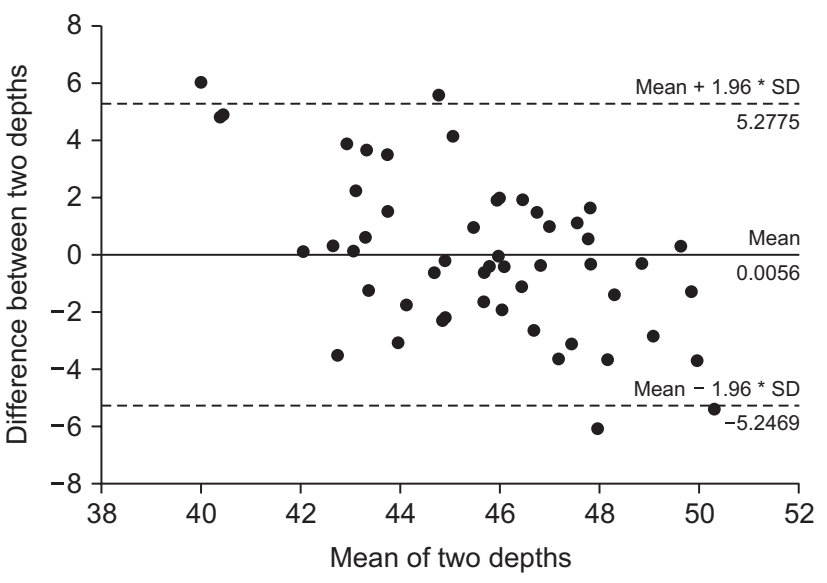

Fig. 2. Bland-Altman plot showing actual PICC depth compared to the depth predicted by the simplified formula; $25+0.5 \times$ (clavicle length $)+$ $0.6 \times$ (sternum length). PICC: peripherally inserted central catheter.

showing that the model using Distances C and D as variables was the optimal model.

Ultimately a regression formula with greatest predictive ability was determined to be $25.3+0.5 \times($ Distance $C)+0.6 \times$ (Distance D) that yielded an $R^{2}$ value of 0.3 . Based on these results, a simplified formula using the patient's clavicle and sternum lengths in centimeters was developed to place PICC catheter tip at the carinal bifurcation, which was expressed as $25.0+0.5 \times$ (clavicle length) $+0.6 \times$ (sternum length). Fig. 2 shows the Bland-Altman plot for actual PICC depths and optimal depths predicted by the simplified formula.

\section{Discussion}

In this study, the lengths from the middle of patients' elbow creases to their carina bifurcations as determined by fluoroscopy during PICC insertions were recorded and used for a formula formation that estimates the proper tip position of the PICC based on anatomic landmarks along its passage. The formula, $25.3+0.5 \times($ Distance $C)+0.6 \times($ Distance $D)$, for determining PICC depth based on the lengths of anatomical landmarks was determined by regression analysis. The purpose of the study ultimately was to develop a simple formula that could be easily applied in clinical settings, so the formula was simplified to 25.0 $+0.5 \times$ (clavicle length) $+0.6 \times($ sternum length $)$ as clavicle and sternum lengths correlated most closely to distances C and D, respectively.

Placing the catheter tip at a proper depth is important for both central venous catheterization and PICC insertion. There are several methods to identify the location of catheter tip [3-7], but the level of carinal bifurcation is a suitable location [16]. Therefore, PICC insertion with fluoroscopic guidance can fix the catheter tip to the appropriate depth during the procedure 
[14]. As an alternative, PICC is often inserted according to the experience of the practitioner then, the chest X-ray is performed afterwards to adjust the proper depth $[15,18]$. Therefore, with an appropriate guideline, it will be possible to reduce various problems from re-adjusting the position of PICC such as patient discomfort, catheter contamination, malfunction, and re-suture.

There has been little research on the appropriate PICC depth, so it is difficult to compare it directly to other procedures. However, central venous catheters follow similar guidelines in that the length of the sternal body is the critical factor in determining the central venous catheter depth, regardless of whether the catheter is inserted into the internal jugular or subclavian vein [19]. Table 2 shows how a patient's height, upper arm length, lower arm length, and sternal body length are associated with each other. As sternal length showed the largest correlation coefficient, it was selected over patient height or arm lengths. A previous retrospective study developed a formula using the patient height, but its accuracy was relatively low [20].

For PICC, the depth for actual insertion must incorporate the distance between the insertion location and elbow crease. For example, if insertion site is $3 \mathrm{~cm}$ above the elbow crease, $3 \mathrm{~cm}$ should be deleted from the depth recommended by the guideline. Conversely, if it was inserted $2 \mathrm{~cm}$ below the elbow crease, $2 \mathrm{~cm}$ must be added to the recommended depth.

In a previous study in which PICC insertion was performed on pediatric patients, catheter tip position changed according to the patient's arm position when the PICC was inserted, regardless of where it was inserted [19]. In this study, patients' arms were held out at 90 degree to their abdomens with straight elbows during PICC insertion. This arm positioning must be used when applying this guideline.

This study has some limitations. The golden standard for identifying catheter tip location is echocardiography [21]. However, we performed fluoroscopy and referenced the correct depth by the carina bifurcation as a surrogate, which is known to be closely associated with the location of cavoatrial junction. The number of participants was limited, which reduced the reliability of the simplified formula. This study included only patients with PICC insertions in their right arms that were held straight out perpendicular to their bodies. While a previous study of pediatric patients showed that there was no significant difference in insertion depth between the left and right arms [19], guidelines for insertion in the left arm of adults is also needed.

In conclusion, the formula proposed for proper depth of the adult, $25.0+0.5 \times$ (clavicle length) $+0.6 \times$ (sternum length) for PICC insertion can be used to place the tip at the carina bifurcation level. The distance from elbow crease to catheter insertion point should be added or subtracted to the length generated by this formula.

\section{References}

1. Periard D, Monney P, Waeber G, Zurkinden C, Mazzolai L, Hayoz D, et al. Randomized controlled trial of peripherally inserted central catheters vs. peripheral catheters for middle duration in-hospital intravenous therapy. J Thromb Haemost 2008; 6: 1281-8.

2. Chopra V, Flanders SA, Saint S. The problem with peripherally inserted central catheters. JAMA 2012; 308: 1527-8.

3. McGee WT, Ackerman BL, Rouben LR, Prasad VM, Bandi V, Mallory DL. Accurate placement of central venous catheters: a prospective, randomized, multicenter trial. Crit Care Med 1993; 21: 1118-23.

4. Ezri T, Weisenberg M, Sessler DI, Berkenstadt H, Elias S, Szmuk P, et al. Correct depth of insertion of right internal jugular central venous catheters based on external landmarks: avoiding the right atrium. J Cardiothorac Vasc Anesth 2007; 21: 497-501.

5. Huh J, Yoo SY, Ro YJ, Min SW, Bahk JH, Kim JS. Survey of central venous catheter depth using the carina as a radiologic landmark in ICU patients. Korean J Anesthesiol 2005; 49: 376-80.

6. Na HS, Kim JT, Kim HS, Bahk JH, Kim CS, Kim SD. Practical anatomic landmarks for determining the insertion depth of central venous catheter in paediatric patients. Br J Anaesth 2009; 102: 820-3.

7. Kim H, Jeong CH, Byon HJ, Shin HK, Yun TJ, Lee JH, et al. Predicting the optimal depth of left-sided central venous catheters in children. Anaesthesia 2013; 68: 1033-7.

8. Jung CW, Lim YJ, Kim SD. Estimation of the optimal depth of subclavian catheterizations in pediatric patients. Korean J Anesthesiol 1999; 37: 426-30.

9. Dane TE, King EG. Fatal cardiac tamponade and other mechanical complications of central venous catheters. Br J Surg 1975; 62: 6-10.

10. Iberti TJ, Katz LB, Reiner MA, Brownie T, Kwun KB. Hydrothorax as a late complication of central venous indwelling catheters. Surgery 1983; 94: 842-6.

11. Dailey RH. Late vascular perforations by CVP catheter tips. J Emerg Med 1988; 6: 137-40.

12. Krauss D, Schmidt GA. Cardiac tamponade and contralateral hemothorax after subclavian vein catheterization. Chest 1991; 99: 517-8.

13. Duntley P, Siever J, Korwes ML, Harpel K, Heffner JE. Vascular erosion by central venous catheters. Clinical features and outcome. Chest 1992; 101: 1633-8.

14. Cardella JF, Cardella K, Bacci N, Fox PS, Post JH. Cumulative experience with 1,273 peripherally inserted central catheters at a single 
institution. J Vasc Interv Radiol 1996; 7: 5-13.

15. Neuman ML, Murphy BD, Rosen MP. Bedside placement of peripherally inserted central catheters: a cost-effectiveness analysis. Radiology 1998; 206: 423-8.

16. Schuster M, Nave H, Piepenbrock S, Pabst R, Panning B. The carina as a landmark in central venous catheter placement. Br J Anaesth 2000; 85: 192-4.

17. Shin TJ, Yoon SJ, Park C, Kim CS, Kim SD. The optimal depth of central venous catheter by using transesophageal echocardiography for pediatric patients. Korean J Anesthesiol 2005; 48: S11-4.

18. Venkatesan T, Sen N, Korula PJ, Surendrababu NR, Raj JP, John P, et al. Blind placements of peripherally inserted antecubital central catheters: initial catheter tip position in relation to carina. Br J Anaesth 2007; 98: 83-8.

19. Connolly B, Amaral J, Walsh S, Temple M, Chait P, Stephens D. Influence of arm movement on central tip location of peripherally inserted central catheters (PICCs). Pediatr Radiol 2006; 36: 845-50.

20. Jeon EY, Koh SH, Lee IJ, Ha HI, Park BJ. Useful equation for proper estimate of left side peripherally inserted central venous catheter length in relation to the height. J Vasc Access 2015; 16: 42-6.

21. Andropoulos DB, Bent ST, Skjonsby B, Stayer SA. The optimal length of insertion of central venous catheters for pediatric patients. Anesth Analg 2001; 93: 883-6. 